\title{
OBJECT-BASED IMAGE ANALYSIS FOR HISTORIC MAPS CLASSIFICATION
}

\author{
P. Zatelli ${ }^{1}$, S. Gobbi ${ }^{1,2,3 *}$ C. Tattoni ${ }^{1}$, N. La Porta ${ }^{2,3,4}$, M. Ciolli ${ }^{1}$ \\ ${ }^{1}$ University of Trento, Dep. of Civil, Environmental and Mechanical engeneering, Trento, Italy - \\ (paolo.zatelli, stefano.gobbi, clara.tattoni, marco.ciolli)@unitn.it \\ ${ }^{2}$ Fondazione Edmund Mach, San Michele a/Adige, Trento, Italy \\ ${ }^{3}$ The EFI Project Centre on Mountain Forests (MOUNTFOR), San Michele a/Adige, Trento, Italy \\ ${ }^{4}$ IASMA Research and Innovation Centre, Fondazione Edmund Mach, San Michele a/Adige, Trento, Italy - nicola.laporta@ fmach.it
}

Commission IV, WG IV/4

KEY WORDS: Heritage maps, classification, OBIA, image filtering, land use, cadaster

\begin{abstract}
:
Heritage maps represent fundamental information for the study of the evolution of a region, especially in terms of landscape and ecologic features. Historical maps present two kinds of hurdle before they can be used in a modern GIS: they must be geometrically corrected to correspond to the datum in use and they must be classified to exploit the information they contain. This study deals the latter problem: the Historical Cadaster Map, created between 1851 and 1861, for the Trentino region in the North of Italy is available as a collection of maps in the ETRS89/UTM 32N datum. The map is a high resolution scan (230 DPI, 24 bit) of the original map and has been used in several ecological studies, since it provides detailed information not only about land property but also about land use. In the past the cadaster map has been manually digitized and for each area a set of attributes has been recorded. Since this approach is time consuming and prone to errors, automatic and semi-automatic procedures have been tested. Traditional image classification techniques, such as maximum likelihood classification, supervised or un-supervised, pixelwise and contextual, do not provide satisfactory results for many reasons: map colors are very variable within the same area, symbols and characters are used to identify cadaster parcels and locations, lines, drawn by hand on the original map, have variable thickness and colors. The availability of FOSS tools for the Object-based Image Analysis (OBIA) has made possible the application of this technique to the cadaster map. This paper describes the use of GRASS GIS and R for the implementation of the OBIA approach for the supervised classification of the historic cadaster map. It describes the determination of the optimal segments, the choice of their attributes and relevant statistics, and their classification. The result has been evaluated with respect to a manually digitized map using Cohens Kappa and the analysis of the confusion matrix. The result of the OBIA classification has also been compared to the classification of the same map using maximum likelihood classification, un-supervised and supervised, both pixelwise and contextual. The OBIA approach has provided very satisfactory results with the ability to automatically remove the background and symbols and characters, creating a ready to be used classified map. This study highlights the effectiveness of the OBIA processing chain available in the FOSS4G ecosystem, and in particular the added value of the interoperability between GRASS GIS and R.
\end{abstract}

\section{INTRODUCTION}

The availability of long time series for the description of the evolution the landscape and of the ecologic features of a region is fundamental (Antrop, 2005) (Tattoni et al., 2017). While the analysis of historical maps to extend the time span of available time series has been investigated for some time (Haase et al., 2007), the number of studies exploiting this possibility is still limited (Dittrich et al., 2017).

There are several reasons for this: on one hand suitable historical maps are uncommon, on the other the use of these maps for use in a Geographic Information System (GIS) system requires substantial pre processing. For historical maps to be suitable for landscape and ecological studies, they must homogeneously cover the study area and feature information about land use/land cover (LULC) (Cantiani et al., 2016).

These two conditions are seldom met, as most historical maps have been created for specific purposes and do not carry this type of information. Even when a suitable map is available, the pre processing needed to prepare the map for its use in a GIS can be challenging. In fact, maps must be digitized, reprojected in the reference system and projection in use, and classified into LULC classes of interest.

\footnotetext{
* Corresponding author
}

This paper describes a new approach for the semi-automatic classification of historical maps and the automatic removal of unwanted artifacts, such as text and symbols on the map. This approach combines R (R Core Team, 2019) and GRASS GIS (GRASS Development Team, 2019) capabilities to digitize and classify an historical map using Object-based Image Analysis (OBIA). The classification is followed by a tailored filtering process to remove text strings and symbols from the map.

\section{MATERIALS AND METHODS}

The main purpose of this research is to develop a semi-automatic procedure for the classification and digitalization of historical maps. The procedure has been set up for the Historical Cadaster Map of the Trentino region and tested on this map.

Historical cadaster maps have already been used to describe the evolution of landscape and ecological features in other parts of Italy (Agnoletti, 2007) and Europe (Forejt et al., 2018), (Skalo , Engstov, 2010), but the maps have been always digitized by manually creating vector areas in a GIS. Even the same Historical Cadaster Map of the Trentino region has already been used after manual digitalization (Tattoni et al., 2010) (Ciolli et al., 2012), but only on a small region. Given the large 
area covered by the cadaster map, this approach would be time consuming and prone to errors.

\subsection{Historic Cadaster Map}

The Historical Cadaster Map for the Province of Trento has been created as part of the cadaster map of the Austrian Empire, under the Royal Imperial Patents of the 23rd of December, 1817 by Francis I, the first Emperor of Austria (Buffoni et al., 2016). The reference network has been established in the Trentino region between 1851 and 1855 (Servizio Catasto della Provincia Autonoma di Trento, 2019a), with the complete survey carried out between 1855 and 1861 (Buffoni et al., 2016).

The purpose of this map is not only the geometric description of objects and property boundaries, but also the representation of LULC, with particular attention to different agricultural uses, and natural boundaries. This maps is therefore suitable for the analysis of landscape and ecologic features, since LULC patches can be recognized.

The original maps, 52,68 x 65,85 cm wide, covering 288 ha, have been digitized at 230 DPI, 24 bit color depth, and the complete map for the Trentino region is available as a set of JPEG images (Servizio Catasto della Provincia Autonoma di Trento, 2019b). Each image contains one of the 13297 original single map sheets or one of the map mosaics; files have dimension between 5 and $8 \mathrm{Mb}$ each. They are accompanied by a JGW world file with coordinates in the ETRS89/UTM $32 \mathrm{~N}$ (EPSG 25832) datum. Additional material, such as surveying maps, cadastral borders descriptions, pictorial and geometric drawings, cadastral monographies and triangulation points are available on a dedicated web site (Servizio Catasto della Provincia Autonoma di Trento, 2019a).

The digital map is available under the Creative Commons Attribution 4.0 license (OPENdata Trentino, 2019).

All tests have been carried out on the map sheets 22_03, 22_04 and 22_05 of the Comune catastale di Cunevo (Cadaster municipality of Cunevo) because they are representative of all the possible combinations of classes and objects sizes. Map sheet 22_05 is shown in figure 1: all successive images and tables refer to this map.

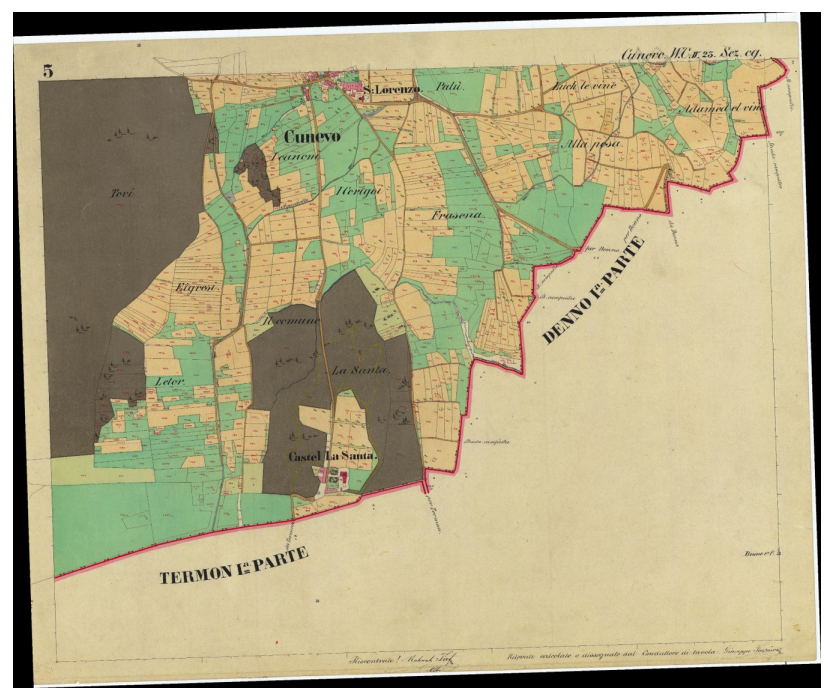

Figure 1. Map sheet 22_05 of the Comune catastale di Cunevo used for the tests.

\subsection{Geo-referentiation}

Digital maps available at (Servizio Catasto della Provincia Autonoma di Trento, 2019b) are already geo-referenced in the ETRS89/UTM $32 \mathrm{~N}$ (EPSG 25832) datum. The geo-referentiation has been carried out by the Servizo Catasto (Cadaster Service) of the Provincia Autonoma di Trento using the four corners of each sheet, whose ETRS89/UTM $32 \mathrm{~N}$ coordinates have been previously determined. Coordinates accuracy is of 5-10 m, but can be improved locally by applying a successive transformation using local Ground Control Points, achieving a 1-2 m accuracy (Revolti, R., 2017). The ground resolution of the map is around $0.32 \mathrm{~m}$.

\subsection{Classification}

The classification of historical maps is usually performed by visual interpretation and manual classification, see e.g. (Statuto et al., 2017) (Forejt et al., 2018). This requires the manual digitalization of each parcel boundary and the input of the parcel number and LULC class. This approach is not only time consuming but also prone to errors:

1. boundary location can be subjective and dependent on the scale/zoom level used during the digitalization

2. errors can be made performing a repetitive task

3. wrong LULC classes can be assigned

4. topology errors are frequent, in particular with overlapping polygons or gaps between them

These errors can be partly removed by adding post-processing steps to the procedure, but an automatic or semi-automatic approach would avoid many of these problems altogether.

The classification of the cadaster historical map has been carried out using the image classification methods available in GRASS:

\section{Maximum Likehood Classification (MLC)}
(a) unsupervised
(b) supervised
(c) supervised contextual

2. Object-based Image Analysis (OBIA)

The first approach has already been tested for other maps (Ferretti et al., 2018) with unsatisfactory results and is used in this study only as a reference to evaluate the classification step of OBIA. For this reason it will not be described in detail. The output maps of the unsupervised MLC, supervised MLC and supervised contextual MLC are shown in figures 2, 3 and 4 respectively for the LULC classes of table 1; colors for classes have been chosen to mimic the colors on the original map. Note that for unsupervised MLC it has not been possible to find a one to one correspondence between classes on the map and LULC classes of table 1. Classes 6 (Black strings and symbols) and 10 (Red strings and symbols) are used to identify text and symbols on the map and will be filtered in the final map.

An assessment of the performance of MLC is reported in table 3. 


\begin{tabular}{|c|l|}
\hline Class & Description \\
\hline 1 & Forest \\
2 & Crop land \\
3 & Meadow \\
4 & Pasture \\
5 & Buildings \\
6 & Black strings and symbols \\
7 & Roads \\
8 & Boundary lines \\
9 & Gardens and courts \\
10 & Red strings and symbols \\
\hline
\end{tabular}

Table 1. LULC classes used for MLC

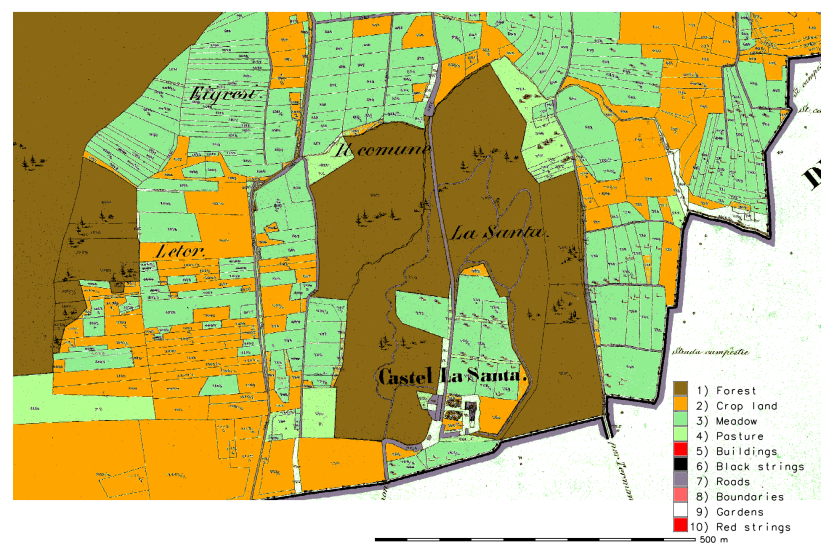

Figure 2. Classified map for unsupervised MLC particular.

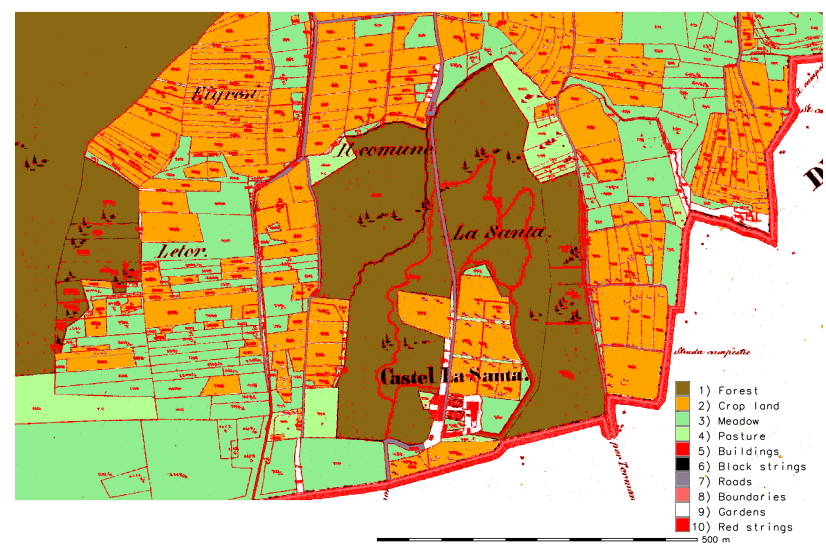

Figure 3. Classified map for supervised MLC - particular.

\subsection{Object-based Image Analysis}

OBIA consists in the creation on the image of "segments" (homogeneous areas) whose radiometric and geometric features are used to classify them. This approach is available in GRASS (Grippa et al., 2017) though a set of add-on modules. The procedure is accomplished in two steps: segment creation and classification.

2.4.1 Segmentation Segment creation in GRASS is performed by the i.segment module. The region growing image segmentation approach has been used in this study, but mean shift image segmentation is also available using the same

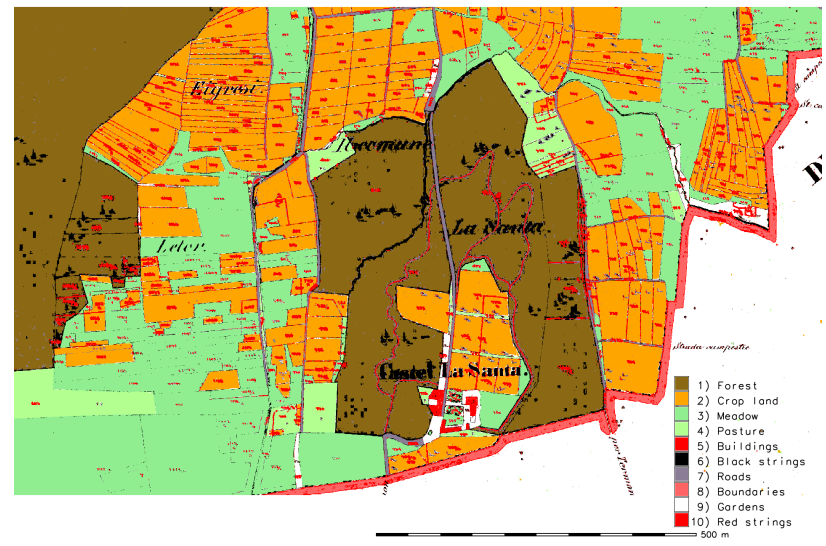

Figure 4. Classified map for contextual supervised MLC particular.

module.

The region growing algorithm is controlled by two parameters: a similarity threshold, which controls the maximum difference of cells in a segment, and the minimum number of cells in a segment, smaller segments are merged. Both parameters have a fundamental role in determining the outcome of the segments creation and therefore of the map classification. The minimum number of cells in a segment can be set by determining the smallest object that must be classified on the map, while the similarity threshold can be chosen empirically, trying different values and evaluating the result.

GRASS implements another approach in the i.segment.uspo add-on module (Grippa et al., 2017), which performs an unsupervised segmentation parameter optimization (USPO), indicating the best segmentation as the one maximizing the $F$ function described in (Johnson et al., 2015). Tests for the cadaster map indicate optimum values for the threshold of 0.05 and a minimum number of cells in a segment of 10 , corresponding to a value $F=1.827$. However, an analysis of the size of the smallest objects on the map has suggested to increase the minimum number of cells to 25 , while the threshold has been raised to 0.1 to avoid the creation of a large number of spurious small segments mostly due to artifacts imputable to JPEG compression. Higher threshold values would hinder the correct classification of small letters and symbols.

A particular of the segmentation of the test map for a threshold value of 0.1 and a minimum number of cells in a segment of 25 is shown in figure 5 .

2.4.2 Classification Segments have been classified into the 12 LULC classes (table 2) on the map using the GRASS v.class.mlR add-on module. With respect to the MLC tests, 2 additional classes ("Black lines" and "Red lines") have been used (table 2), to account for the fact that OBIA relies also on the shape of the objects (segments) for the classification: letters and symbols compactness is very different from compactness of lines.

This module performs the classification using the direct connection between GRASS and R, exploiting the capabilities of R's Caret library. All the available classifiers, Support Vector Machine (with a radial kernel) (SVMradial), Random Forest (RF), Recursive partitioning (Rpart), and k-Nearest Neighbors $(\mathrm{kNN})$ have been used. All the available voting systems, 


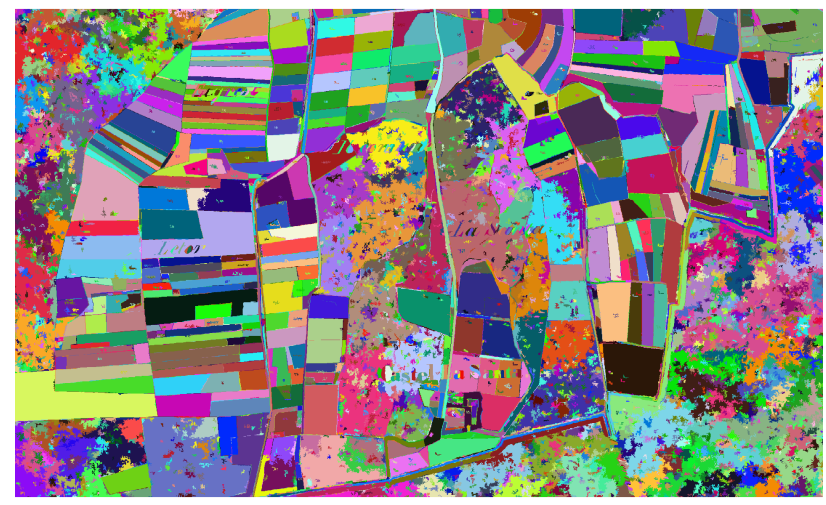

Figure 5. Segments for threshold value of 0.1 and minimum number of cells in a segment of 25 - particular.

\begin{tabular}{|c|l|}
\hline Class & Description \\
\hline 1 & Forest \\
2 & Crop land \\
3 & Meadows \\
4 & Pasture \\
5 & Buildings \\
6 & Black strings and symbols \\
7 & Roads \\
8 & Boundary lines \\
9 & Gardens and courts \\
10 & Red strings and symbols \\
11 & Black lines \\
12 & Red lines \\
\hline
\end{tabular}

Table 2. LULC classes used for OBIA classification.

Simple Majority Vote (SMV), Simple Weighted Vote (SWV), Best Worst Weighted Vote (BWWV) and Quadratic Best Worst Weighted Vote (QBWWV) have also been used.

A training map containing 331 sample points (figure 6) and 12 LULC classes (table 2) has been created for training. LULC classes are assigned to the segments they fall into after the segmentation by joining the tables of the two vector maps, using the segment id as join field.

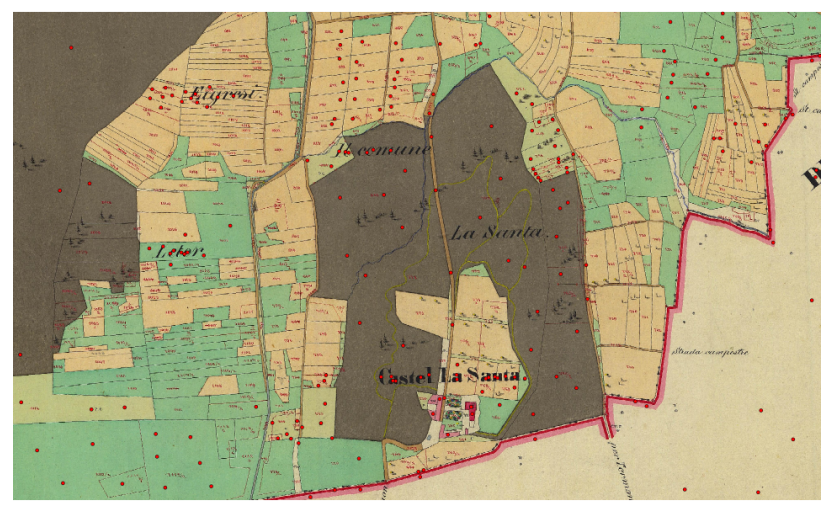

Figure 6. Training points (red dots) - particular.

The output of the classification using the SWV voting system is shown in figure 7.

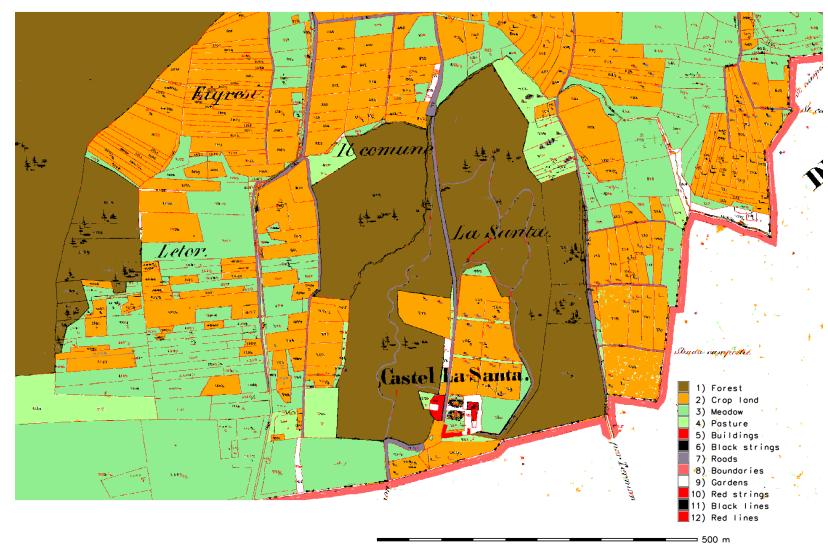

Figure 7. Classified map for OBIA and BWWV particular.

\subsection{Text and symbols removal}

One of the main obstacles of the application of automatic classification techniques to maps is the presence of text string and symbols, which must be deleted in the classified map, where the user is interested in LULC.

For this reason a new GRASS add-on module, rreplace, has been written, which substitutes the values of pixels of a given category with values of the surrounding pixels and stores the output in a new raster map. Pixels with other categories are left untouched.

The module iteratively applies a low-pass filter to the pixels with the category to remove, assigning the mode of the category of the neighbor pixels. The user controls the size of the neighborhood: large values can speed the process considerably but can also lead to unwanted effects where pixels with different categories are mixed, small values of the neighborhood size require a larger number of iterations and longer processing times, but provide better results when categories are mixed. The module can also create a MPEG animation of the iterative process.

The module is currently being tested and will be made available as a GRASS add-on module under the GNU General Public License $(\geq \mathrm{v} 2)$ used for GRASS source code.

The application of this module to the classified images using unsupervised MLC, supervised MLC, supervised contextual MLC and OBIA with the BWWV voter is shown in figures 8 , 9,10 and 11 .

The method is effective, as long as segments containing a text string or a symbol are correctly classified. Tests are under way to determine the best value for the neighborhood size for the filter depending on the image.

\section{RESULTS}

To check the results of the classification, 96 areas have been manually digitized on the map. Eight areas have been created for each of the 12 categories: 4 areas of the first 10 categories have been used for training the supervised and supervised contextual MLC, the other 4 have been used to evaluate the overall accuracy and Cohen's Kappa parameter. Areas of the last 2 categories have been used to assess the overall accuracy and Cohen's Kappa parameter for the OBIA output maps. 


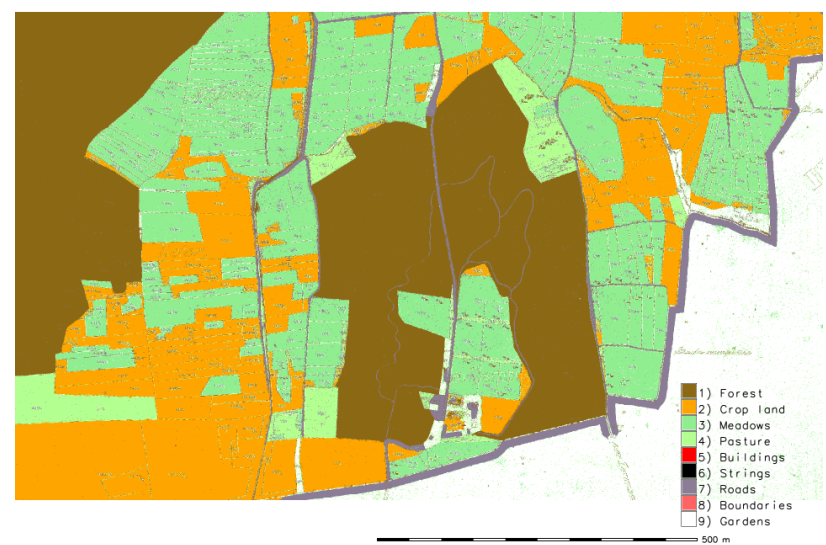

Figure 8. Classified map for unsupervised MLC after text and symbols removal - particular.

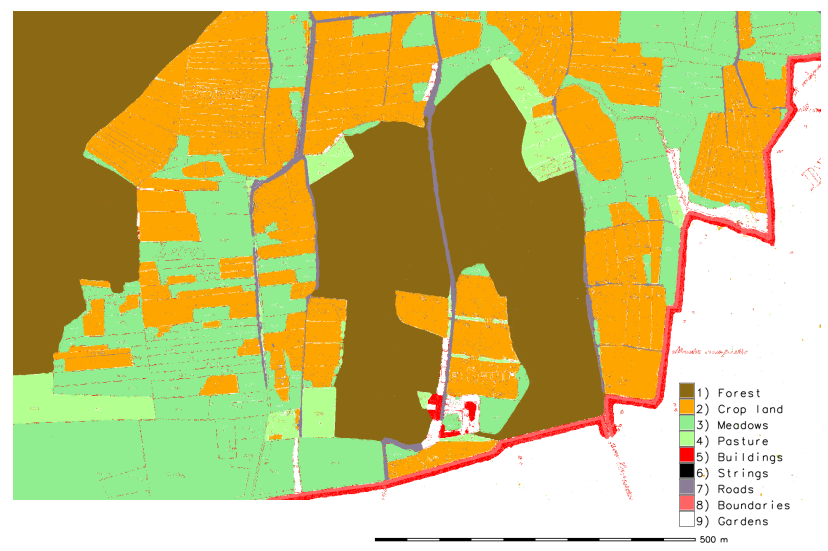

Figure 9. Classified map for supervised MLC after text and symbols removal - particular.

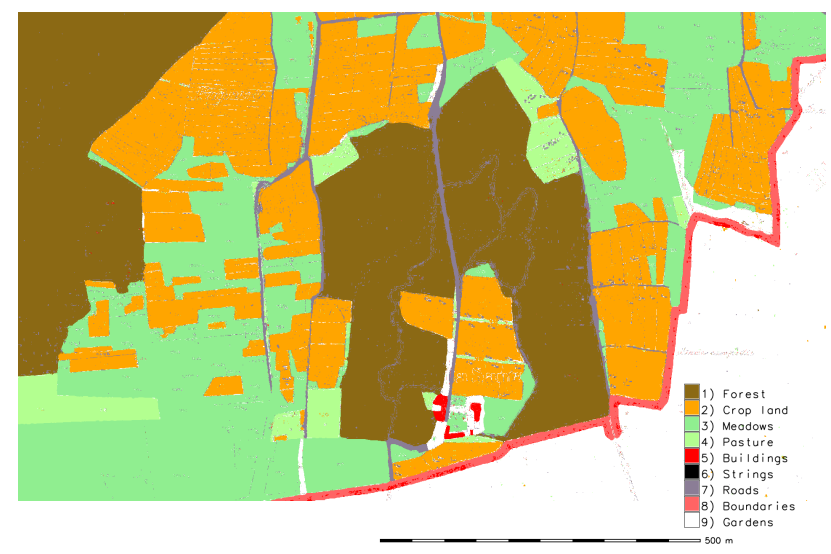

Figure 10. Classified map for contextual supervised MLC after text and symbols removal - particular.

Results for the 3 MLC classifiers and 4 voting systems for OBIA are reported in tab 3.

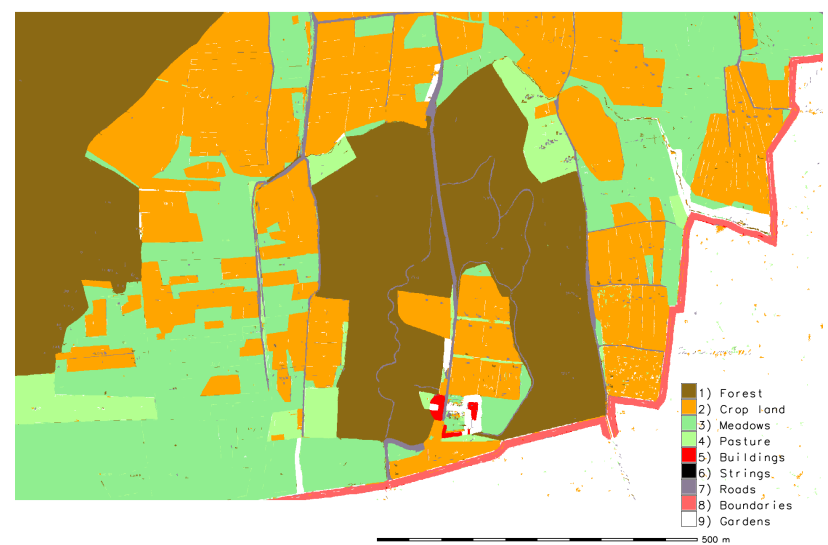

Figure 11. Classified map for OBIA and BWWV after text and symbols removal - particular.

\begin{tabular}{cccc} 
& Classifier & OA & Kappa \\
\hline \multirow{2}{*}{ MLC } & Unsupervised & $67.9 \%$ & 0.473 \\
& Supervised & $97.6 \%$ & 0.960 \\
& Supervised Contextual & $98.7 \%$ & 0.978 \\
\hline \multirow{2}{*}{ OBIA } & SMV & $98.7 \%$ & 0.978 \\
& SWV & $99.0 \%$ & 0.983 \\
& BWWV & $99.0 \%$ & 0.984 \\
& QBWWV & $99.0 \%$ & 0.984 \\
\hline
\end{tabular}

Table 3. Overall accuracy and Coen's Kappa for different classifiers. OA: Overall accuracy. MLC: Maximum Likehood Classification. OBIA: Object-based Image Analysis. SMV: Simple Majority Vote. SWV: Simple Weighted Vote. BWWV: Best Worst Weighted Vote.

QBWWV: Quadratic Best Worst Weighted Vote.

Table 3 shows how OBIA using BWWV and QBWWV voters achieves the highest values of overall accuracy and Cohen's Kappa. LULC maps are also more uniform, while all maps from MLC contain fragmented areas. Note that the last two voting systems, Best Worst Weighted Vote (BWWV) and Quadratic Best Worst Weighted Vote (QBWWV), assign exactly the same votes, therefore the resulting classified map, the overall accuracy and Kappa are the same.

The overall accuracy and Cohen's Kappa parameter have been evaluated again after strings and lines removal (table 4), having removed classess 6 (Black strings and symbols), 10 (Red strings and symbols), 11 (Black lines) and 12 (Red lines).

Values for maps from OBIA classifiers do not change, because no segment in the remainig 9 classes were (mis)classified as one of the 4 removed classes: 6 (Black strings and symbols), 10 (Red strings and symbols), 11 (Black lines) and 12 (Red lines). As expected, Kappa and OA improve slightly for MLC, mainly because of the removal of pixels of classes 6 (Black strings and symbols) and 10 (Red strings and symbols) in the test areas.

Confusion matrixes for Supervised Contextual MLC and OBIA Best Worst Weighted Vote (BWWV) classifiers are reported in tables 5 and 6.

Tables 5 and 6 show that both Supervised Contextual MLC and OBIA are able to correctly classify "Forst","Meadows", "Pasture" and "Boundaries" classes, with a slightly better result for Supervised Contextual MLC for the "Pasture" 


\begin{tabular}{cccc} 
& Classifier & OA & Kappa \\
\hline \multirow{3}{*}{ MLC } & Unsupervised & $68.0 \%$ & 0.471 \\
& Supervised & $98.0 \%$ & 0.968 \\
& Supervised Contextual & $99.5 \%$ & 0.991 \\
\hline \multirow{2}{*}{ OBIA } & SMV & $98.7 \%$ & 0.978 \\
& SWV & $99.0 \%$ & 0.983 \\
& BWWV & $99.0 \%$ & 0.984 \\
& QBWWV & $99.0 \%$ & 0.984
\end{tabular}

Table 4. Overall accuracy and Coen's Kappa for different classifiers after strings and lines removal. OA: Overall accuracy. MLC: Maximum Likehood Classification. OBIA: Object-based Image Analysis. SMV: Simple Majority Vote. SWV: Simple Weighted Vote. BWWV: Best Worst Weighted Vote. QBWWV: Quadratic Best Worst Weighted Vote.

class (99.99\% vs $98.44 \%$ accuracy) and for OBIA for the "Boundaries" class (100\% vs $99.76 \%$ accuracy). Supervised Contextual MLC outperforms OBIA for the "Crop land" class, $99.98 \%$ vs $90.3 \%$ accuracy: this is mostly due to one single parcel of the "Gardens" class (figure 12), which has been misclassifed because it has been made part of a larger segment correctly classified as "Crop land" (figure 13).

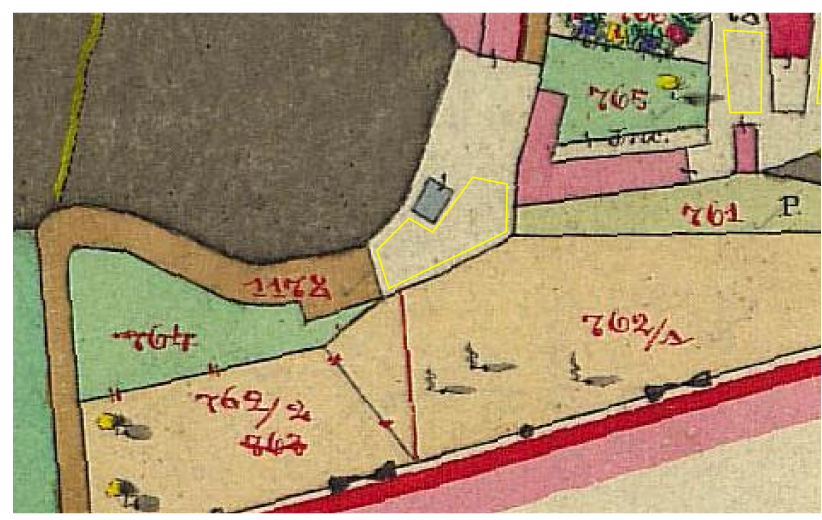

Figure 12. Garden parcel, in the center in light grey, containing a darker grey rectangle, which is misclassified. The extension of one of the check area for the "Gardens" class is shown in yelllow.

OBIA performance clearly surpass Supervised Contextual MLC for the "Buildings" class, $98.43 \%$ vs $55.89 \%$ accuracy, where the color on the map, red, is the same as that used for "Boundaries": OBIA classifiers are able to discriminate using the geometric features of the segments, whereas Supervised Contextual MLC uses only pixel values and neighborhood classes. A similar situation occours for the "Road" class, where taking into account geometric features allows OBIA to reach a $98.56 \%$ accuracy, while similar colors lead to the misclassification of $6.71 \%$ of the "Road"surface as "Forest" for the Supervised Contextual MLC, limiting its accuracy to $92.29 \%$.

OBIA classifiers incorrectly identify $7.16 \%$ of the area of the "Boundaries" class as belonging to the "Gardens" class: some segments are misclassified as "Gardens" because the JPEG compression adds a line of lighter colored pixels, whose color is very similar to color for pixels the "Gardens" class, between the two tones of red of the boundary line (figure 14).

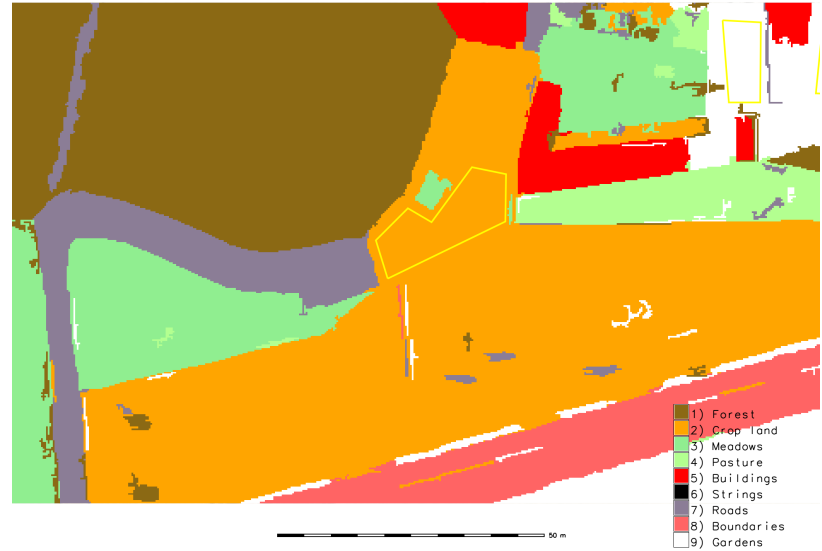

Figure 13. Classified segments for BWWV voter, the garden parcel of figure 12 is misclassified as "Crop land" beacuse it has been merged in a single segment with crop land parcels. The extension of one of the check area for the "Gardens" class is shown in yelllow.

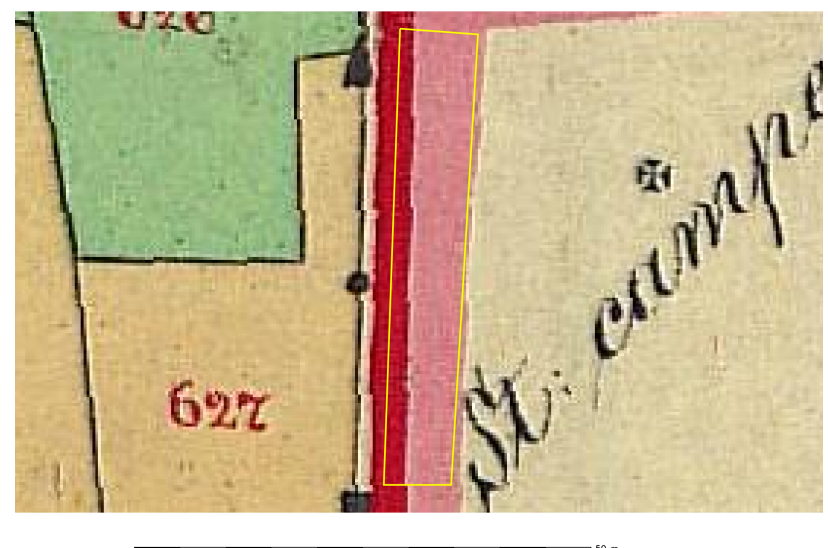

Figure 14. Light tones between two red tones of the boundary line. The extension of one of the check area for the "Boundaries" class is shown in yelllow.

Many misclassifications are due to artifacts introduced by the JPEG compression, the access to the original uncompressed images is currently under negotiation. The application of the procedure to the original images should enhance the result, but tests must be carried out to quantify the benefit of using images encoded in a loseless format.

\section{CONCLUSIONS}

The OBIA approach has proved effective for the semi-automatic digitalization and classification of the Historical Cadaster Map for the Province of Trento.

Special care must be used when choosing the training points, in particular all LULC must be sampled. For the correct classification of text and symbols, it is fundamental to place some training points in the space inside the characters, such as the inner space for the "o" letter, and the symbol drawings, because the classifier must be trained to recognize segments in 


\begin{tabular}{|c|c|c|c|c|c|c|c|c|c|}
\hline & & & \multicolumn{7}{|c|}{ Reference } \\
\hline & Classes & Forest & Crop land & Meadows & Pasture & Buildings & Roads & Boundaries & Gardens \\
\hline & Forest & 100 & 0 & 0 & 0 & 0 & 6.71 & 0 & 0.06 \\
\hline & Crop land & 0 & 99.98 & 0 & 0 & 0 & 0.87 & 0 & 0 \\
\hline Supervised & Meadows & 0 & 0 & 100 & 0 & 0 & 0 & 0 & 0 \\
\hline Contextual & Pasture & 0 & 0 & 0 & 99.99 & 0 & 0 & 0 & 0.10 \\
\hline MLC & Buildings & 0 & 0 & 0 & 0 & 55.89 & 0 & 0.24 & 0 \\
\hline & Roads & 0 & 0.02 & 0 & 0 & 0 & 92.29 & 0 & 0.02 \\
\hline & Boundaries & 0 & 0 & 0 & 0 & 44.11 & 0 & 99.76 & 0 \\
\hline & Gardens & 0 & 0 & 0 & 0.01 & 0 & 0.12 & 0 & 99.83 \\
\hline
\end{tabular}

Table 5. Confusion matrix for Supervised Contextual MLC. Values are percentages of the reference test set (column-based normalization), producer accuracy can be read on diagonal values.

\begin{tabular}{ccccccccccc}
\hline & Classes & Forest & Crop land & Meadows & Pasture & Buildings & Roads & Boundaries & Gardens \\
\hline & Forest & 99.97 & 0 & 0 & 0 & 0 & 1.09 & 0 & 0 \\
& Crop land & 0 & 90.63 & 0 & 1.56 & 0 & 0 & 0 & 2.43 \\
OBIA & Meadows & 0.02 & 0 & 100 & 0 & 0 & 0 & 0 & 0 \\
BWWV & Pasture & 0 & 0 & 0 & 98.44 & 0 & 0 & 0 & 1.38 \\
& Buildings & 0 & 0 & 0 & 0 & 98.43 & 0.35 & 0 & 0 \\
& Roads & 0.01 & 0 & 0 & 0 & 0 & 98.56 & 0 & 0 \\
& Boundaries & 0 & 1.05 & 0 & 0 & 1.57 & 0 & 100 & 7.16 \\
& Gardens & 0 & 8.32 & 0 & 0 & 0 & 0 & 0 & 89.02 \\
\hline
\end{tabular}

Table 6. Confusion matrix for OBIA Best Worst Weighted Vote (BWWV). Values are percentages of the reference test set (column-based normalization), producer accuracy can be read on diagonal values.

these spaces as not belonging to the characters and symbols class. At the same time, the similarity threshold and the minimum number of cells in a segment must be carefully chosen to balance the need of properly classifying text with the aim of avoiding the creation of a large number of small segments which can undermine the classification step and lead to fragmented maps.

The procedure for text strings and symbols removal is operative and very simple to apply, thanks to the creation of a dedicated GRASS add-on module, which will be made available after the current testing phase.

One of the advantages of OBIA is the possibility of creating a training map of points, instead of areas as for the supervised MLC, simplifying the process. Moreover, the training map created for a map sheet or image can be usually applied to other map sheets or images of the same dataset, with a clear advantage, especially or large datasets. In the case of the cadaster historical map for the Province of Trento this is a huge asset, as the dataset contains 13297 map sheets and its classification can be performed in a batch process.

The use of GRASS GIS (GRASS Development Team, 2019) provides a clear advantage for the possibility of rapid development of new procedures and modules (Preatoni et al., 2012), its direct connection with $\mathrm{R}$, and the ease of the dissemination of the results, in particular for educational purposes (Ciolli et al., 2017).

The digitalization of the Historical Cadaster Map of the Trentino region is part of a wider project (Gobbi et al., 2019) which aims to build a dataset containing a large number of digital maps, created from historical maps and historical aereal images (Gobbi et al., 2018), covering a long time span for the Trentino region, as a base for the study of the landscape and ecologic evolution of the area. In particular, the application of this technique to the 1936 Italian Kingdom Forest Map (Ferretti et al., 2018) is planned in the next future.

\section{ACKNOWLEDGMENTS}

The cadaster historical map for the Province of Trento has been made available by the Servizio Catasto della Provincia Autonoma di Trento, under the Creative Commons Attribution 4.0 license.

\section{REFERENCES}

Agnoletti, M., 2007. The degradation of traditional landscape in a mountain area of Tuscany during the 19th and 20th centuries: Implications for biodiversity and sustainable management. Forest Ecology and Management, 249, 5 - 17. doi.org/10.1016/j.foreco.2007.05.032.

Antrop, M., 2005. Why landscapes of the past are important for the future. Landscape and Urban Planning, 70, 21-34. doi.org/10.1016/j.landurbplan.2003.10.002.

Buffoni, D., Girardi, S., Revolti, R., Cortese, G., Mastronunzio, M., 2016. Historicalkat. la documentazione catastale trentina d'impianto è open data. Proceedings of the XX Conferenza Nazionale ASITA, 112-117.

Cantiani, M.G., Geitner, C., Haida, C., Maino, F., Tattoni, C., Vettorato, D., Ciolli, M., 2016. Balancing economic development and environmental conservation for a new governance of Alpine areas. Sustainability (Switzerland), 8. doi.org/10.3390/su8080802.

Ciolli, M., Federici, B., Ferrando, I., Marzocchi, R., Sguerso, D., Tattoni, C., Vitti, A., Zatelli, P., 2017. FOSS tools and applications for education in geospatial sciences. ISPRS International Journal of Geo-Information, 6. doi.org/10.3390/ijgi6070225.

Ciolli, M., Tattoni, C., Ferretti, F., 2012. Understanding forest changes to support planning: A fine-scale Markov chain approach. Developments in Environmental Modelling, 25, 355-373. doi.org/10.1016/B978-0-444-59396-2.00021-3. 
Dittrich, A., von Wehrden, H., Abson, D. J., Bartkowski, B., Cord, A. F., Fust, P, Hoyer, C., Kambach, S., Meyer, M. A., Radzeviit, R., Nieto-Romero, M., Seppelt, R., Beckmann, M., 2017. Mapping and analysing historical indicators of ecosystem services in Germany. Ecological Indicators, 75, 101 - 110. doi.org/10.1016/j.ecolind.2016.12.010.

Ferretti, F., Sboarina, C., Tattoni, C., Vitti, A., Zatelli, P., Geri, F., Pompei, E., Ciolli, M., 2018. The 1936 Italian Kingdom Forest Map reviewed: A dataset for landscape and ecological research. Annals of Silvicultural Research, 42, 3-19. doi.org/10.12899/asr-1411.

Forejt, M., Dolej, M., Raka, P., 2018. How reliable is my historical land-use reconstruction? Assessing uncertainties in old cadastral maps. Ecological Indicators, 94, 237 - 245. doi.org/10.1016/j.ecolind.2018.06.053.

Gobbi, S., Cantiani, M.G., Rocchini, D., Zatelli, P., Tattoni, C., Ciolli, M., La Porta, N., 2019. Fine spatial scale modelling of Trentino past forest landscape (trentinoland): a case study of foss application. In preparation.

Gobbi, S., Maimeri, G., Tattoni, C., Cantiani, M.G., Rocchini, D., La Porta, N., Ciolli, M., Zatelli, P., 2018. Orthorectification of a large dataset of historical aerial images: Procedure and precision assessment in an open source environment. International Archives of the Photogrammetry, Remote Sensing and Spatial Information Sciences - ISPRS Archives, 42 4W8, 53-59.

GRASS Development Team, 2019. Geographic Resources Analysis Support System (GRASS GIS) Web Site. http://grass.osgeo.org. Accessed: 2019-04-29.

Grippa, T., Lennert, M., Beaumont, B., Vanhuysse, S., Stephenne, N., Wolff, E., 2017. An open-source semi-automated processing chain for urban object-based classification. Remote Sensing, 9. doi.org/10.3390/rs9040358.

Haase, D., Walz, U., Neubert, M., Rosenberg, M., 2007. Changes to Central European landscapes-Analysing historical maps to approach current environmental issues, examples from Saxony, Central Germany. Land Use Policy, 24, 248-263. doi.org/10.1016/j.landusepol.2005.09.003.

Johnson, B.A., Bragais, M., Endo, I., Magcale-Macandog, D.B., Macandog, P.B.M., 2015. Image segmentation parameter optimization considering within-and between-segment heterogeneity at multiple scale levels: Test case for mapping residential areas using landsat imagery. ISPRS International Journal of Geo-Information, 4, 2292-2305. doi.org/10.3390/ijgi4042292.

OPENdata Trentino, 2019. Historical Cadaster Map of the Trentino region metadata. https://dati.trentino.it/dataset/mappe-storiche-d-impianto.

Accessed: 2019-04-29.

Preatoni, D.G., Tattoni, C., Bisi, F., Masseroni, E., D'Acunto, D., Lunardi, S., Grimod, I., Martinoli, A., Tosi, G., 2012. Open source evaluation of kilometric indexes of abundance. Ecological Informatics, 7, 35-40. doi.org/10.1016/j.ecoinf.2011.07.002.

R Core Team, 2019. R: A Language and Environment for Statistical Computing. R Foundation for Statistical Computing, Vienna, Austria.
Revolti, R., 2017. Produzione e restauro della cartografia catastale in Trentino. https://drive.google.com/file/d/ 0B0abPZ1IrxqdenUwXzB1T2RvV0E/view. Accessed: 2019-04-29.

Servizio Catasto della Provincia Autonoma di Trento, 2019a. HistoricalKat, documentation of the Trentino cadastral archive. http://historicalkat.provincia.tn.it. Accessed: 2019-04-29.

Servizio Catasto della Provincia Autonoma di Trento, 2019b. Mappe storiche di impianto (urmappe). https://www.catastotn.it/mappeStoriche.html. Accessed: 2019-04-29.

Skalo, J., Engstov, B., 2010. Methodology for mapping non-forest wood elements using historic cadastral maps and aerial photographs as a basis for management. Journal of Environmental Management, 91, 831 - 843. doi.org/10.1016/j.jenvman.2009.10.013.

Statuto, D., Cillis, G., Picuno, P., 2017. Using Historical Maps within a GIS to Analyze Two Centuries of Rural Landscape Changes in Southern Italy. Land, 6.

Tattoni, C., Ciolli, M., Ferretti, F., Cantiani, M.G., 2010. Monitoring spatial and temporal pattern of Paneveggio forest (Northern Italy) from 1859 to 2006. IForest, 3, 72-80. doi.org/10.3832/ifor0530-003.

Tattoni, C., Ianni, E., Geneletti, D., Zatelli, P., Ciolli, M., 2017. Landscape changes, traditional ecological knowledge and future scenarios in the Alps: A holistic ecological approach. Science of the Total Environment, 579, 27-36. doi.org/10.1016/j.scitotenv.2016.11.075. 